\title{
Difference Between Delayed Anastomosis and Early Anastomosis in Damage Control Laparotomy Affecting the Infusion Volume and NPWT Output volume: Is Infusion Restriction Necessary in Delayed Anastomosis? A Single-Center Retrospective Analysis
}

Yohta Tanahashi

Iwate Medical University: Iwate Ika Daigaku

Hisaho Sato

Iwate Medical University: Iwate Ika Daigaku

Akiko Kawakami

Iwate Medical University: Iwate Ika Daigaku

Shusaku Sasaki

Iwate Medical University: Iwate Ika Daigaku

Yu nishinari

Iwate Medical University: Iwate Ika Daigaku

Kaoru Ishida

Iwate Medical University: Iwate Ika Daigaku

Masahiro Kojika ( $\nabla$ kojimasa@iwate-med.ac.jp)

Iwate Medical University https://orcid.org/0000-0002-2418-1526

Shigeatsu Endo

Iwate Medical University: Iwate Ika Daigaku

Yoshihiro Inoue

Iwate Medical University: Iwate Ika Daigaku

Akira Sasaki

Iwate Medical University: Iwate Ika Daigaku

Research article

Keywords: delayed anastomosis, NPWT, damage control laparotomy

Posted Date: August 9th, 2021 
DOl: https://doi.org/10.21203/rs.3.rs-772713/v1

License: (c) (1) This work is licensed under a Creative Commons Attribution 4.0 International License. Read Full License

Version of Record: A version of this preprint was published at Trauma Surgery \&amp; Acute Care Open on March 1st, 2022. See the published version at https://doi.org/10.1136/tsaco-2021-000860. 


\section{Abstract}

Background: Delayed anastomosis is a treatment strategy used in damage control laparotomy (DCL).

During temporary abdominal closure (TAC) with DCL, infusion volume, and negative-pressure wound therapy (NPWT) output volume are associated with the success and prognosis of primary fascial closure (PFC). The same may also hold true for anastomosis. The aim of this research is to evaluate whether the difference between early anastomosis and delayed anastomosis in DCL is related to infusion volume and NPWT output volume.

Methods: This single-center retrospective analysis targeted patients managed with TAC during emergency surgery for trauma or intra-abdominal sepsis between January 2011 and December 2019. It included patients who underwent repair/anastomosis/artificial anus construction in the first surgery and patients who underwent intestinal resection in the first surgery followed by delayed anastomosis with no intestinal continuity. The main outcomes were infusion volume, NPWT output volume and complications.

Results: One hundred nine patients who underwent emergency surgery were evaluated. Seventy-three patients were managed with TAC using NPWT. In 16 patients with early anastomosis and 21 patients with delayed anastomosis, there was no difference in the infusion volume $(p=0.2318)$ or NPWT output volume $(p=0.7128) 48$ hours after surgery. Additionally, there was no difference in the occurrence of surgical site infection $(p=0.315)$ and suture failure $(p=0.8428)$. During the second-look surgery after 48 hours, the anastomosis was further postponed for $48 \%$ of the patients who underwent delayed anastomosis. There was no difference in the infusion volume $(p=0.0783)$ up to the second-look surgery between the patients whose delayed anastomosis was postponed and those who underwent delayed anastomosis, but there was a tendency toward a large NPWT output volume $(p=0.024)$ in the postponed delayed anastomosis group. Anastomosis and PFC were achieved for all patients whose delayed anastomosis was postponed.

Conclusions: The presence or absence of anastomosis during TAC management does not affect NPWT output volume. Delayed anastomosis may be managed with the same infusion volume as that used for early anastomosis. There is also the option of postponing anastomosis if the planned delayed anastomosis is complicated.

\section{Trial Registration}

The retrospective protocol of this study was approved by our institutional review board (MH2018-611).

\section{Background}

Delayed anastomosis is a treatment strategy that is incorporated into damage control laparotomy (DCL) according to the physiological indicators and intra-abdominal environments of patients who have no intestinal continuity after undergoing only intestinal resection during initial surgery for hollow viscous injury ( $\mathrm{HVI})$ or mesenteric injury $(\mathrm{MI})[1-6]$. After DCL, the patient is moved quickly to the intensive Care 
Unit for correcting hypothermia, acidosis, coagulative disorders and performing physiological optimization [1, 7-9]. Temporary abdominal closure (TAC) using negative-pressure wound therapy (NPWT) is the general approach used until repeat laparotomy is performed. These DCL treatment strategies are applied not only to trauma but also to intra-abdominal sepsis and are very important treatment strategies [10-12]. However, the decision to perform anastomosis during the first procedure or delay it is complex and must consider the patient's physiological indicators, damage status, contamination status, and other factors.

It has been reported that during primary fascial closure (PFC) after TAC, excessive infusion leads to tissue edema and prevents successful PFC [13-15] and that limited use of the crystalloid infusion protocol improves the success of PFC [16]. For similar reasons, excessive infusion may lead to the development of intestinal edema, which may prevent successful delayed anastomosis or require the construction of an artificial anus to avoid anastomosis. In addition, the NPWT output volume in TAC is an important fluid balance index, and hypoalbuminemia, in which albumin is lost when protein-rich ascites are drained [17], may pose a risk of anastomotic leakage [18-20] and present a disadvantage for the success of delayed anastomosis. However, there is little information regarding the differences and relevance of infusion volume and NPWT output volume during TAC for the management of early anastomosis and delayed anastomosis in DCL.

The aim of this research is to evaluate whether the difference between early anastomosis and delayed anastomosis is related to infusion volume and NPWT output volume in patients who underwent DCL due to trauma and intra-abdominal sepsis.

\section{Methods}

\section{Target}

The protocol of the study was approved by the institutional review board.

This retrospective study was conducted at the Iwate Medical University Critical Care Center from 2011 to 2019 with patients who underwent emergency surgery due to $\mathrm{HVI} / \mathrm{Ml}$ caused by abdominal trauma and patients who underwent emergency surgery for lower digestive tract perforation with diffuse peritonitis and peritoneal contamination in the form of intra-abdominal sepsis. The study included patients who underwent repair/anastomosis/artificial anus construction during the first DCL surgery and were managed with TAC using NPWT and patients who had no intestinal continuity after undergoing intestinal resection during the first surgery and were managed with TAC using NPWT. Clinical data and infusion volume, NPWT output, and urine volume up to 48 hours after surgery were extracted from chart review or clinical records review.

The study excluded patients who died within 48 hours after surgery, patients with solid organ injuries without $\mathrm{HVl}$, patients who underwent nonsurgical treatment (for Ml or intra-abdominal hemorrhage), patients on maintenance dialysis due to chronic renal failure, and patients with iatrogenic injuries. 
Patients with perforated appendicitis and patients who underwent resurgery with DCL were not included in the study.

\section{Treatment Strategy}

\section{Repair, Resection and Artificial Anus Construction}

Treatment was conducted at the discretion of the surgeon and not protocolized according to specific injury or perforation site and morphology or contamination status. Intestinal resection was performed using a stapler. In cases in which delayed anastomosis was selected, the end of the intestinal resection remained stapled, and the intestine was left discontinuous and was directed into the abdominal cavity. No temporary artificial anus was created until anastomosis was performed. For delayed anastomosis, second-look surgery was performed 48 hours after the initial surgery to evaluate whether to restore intestinal continuity or create an artificial anus. Other options included postponing anastomosis with no intestinal continuity and postponing abdominal closure. When TAC was continued, the condition of the intestinal tract was evaluated in a timely manner at the intensive care unit or high-dependency care unit, and the abdomen was closed after anastomosis or artificial anus construction was selected at the discretion of the surgeon. Regarding the anastomosis technique, stapling anastomosis was performed in all cases, and hand-sewn anastomosis was not performed. lleostomy was not performed after anastomosis.

\section{Infusion Resuscitation}

The infusion dose was based on physiological indicators such as heart rate, blood pressure, etc., and the diameter of the inferior vena cava according to ultrasound examination. Infusion up to 48 hours after surgery included basic infusion crystalloid fluid, bolus-administered crystalloid fluid, and albumin preparations. The administration of the albumin preparation was capped at 1 dose of $100 \mathrm{ml} 20 \%$ albumin preparation per day. There was no protocol for bolus administration of crystalloid solution corresponding to the NPWT output amount. Furosemide was not used for up to 48 hours after surgery. When hemodynamics were unstable even after sufficient infusion (systolic blood pressure less than 90 $\mathrm{mmHg}$ ), the administration of $0.05 \mu \mathrm{g} / \mathrm{kg} / \mathrm{min}$ of norepinephrine as a vasopressor was started.

\section{Negative-Pressure Wound Therapy}

NPWT was carried out with a handmade negative pressure system after the abdominal cavity was thoroughly rinsed with saline. The intra-abdominal organs were covered with a sterile vinyl sheet. A $28 \mathrm{Fr}$ gastric catheter was placed on the top of the vinyl sheet, a transparent adhesive film was affixed to the top of the catheter, and TAC was performed with a suction pressure of $-30 \mathrm{cmH} 20$. After that, an elastic band was used to cover the abdomen to prevent the abdominal wall from retreating. During TAC management, sedatives, analgesics, and neuromuscular blocking drugs were administered and controlled based on ventilator monitoring.

The same surgical team performed all steps from diagnosis to surgery to postoperative management. 


\section{Blood Sampling and Assay}

Blood and serum biochemical tests and arterial blood gas analysis were performed before, immediately after and 48 hours after surgery at our central clinical laboratory. The test data were extracted by reviewing the clinical records.

\section{Definitions and Study Outcomes}

Patients were classified as follows. Patients who underwent repair/anastomosis/artificial anus construction during the DCL first surgery and were managed with TAC using NPWT were classified as DCL-early, and patients who had no intestinal continuity after intestinal resection during the first surgery and were managed with TAC using NPWT were classified as DCL-delay. Patients who underwent early anastomosis were classified as DCL-early anastomosis, and patients who underwent delayed anastomosis were classified as DCL-delayed anastomosis. Patients were also grouped according to whether they underwent delayed anastomosis during second-look surgery 48 hours after the initial surgery or their delayed anastomosis was postponed.

The important research results were infusion volume and NPWT output up to 48 hours after the initial surgery with early anastomosis and delayed anastomosis. Secondary results were infusion volume with or without therapeutic intervention or complications, NPWT output, and urine volume. The delayed anastomosis implementation status and frequency of complications were also included. Complications were surgical site infection (SSI), intestinal obstruction, and suture failure.

\section{Statistical Analysis}

All statistical analyses were performed with the statistical package JMP® 11 (SAS Institute Inc., Cary, NC, USA). Continuous variables are expressed as the mean \pm SD. Categorical variables are expressed as frequency $(n, \%)$, as appropriate. Normal distribution of all data was confirmed using the Shapiro-Wilk test. The data were analyzed using the chi-squared test, Wilcoxon rank-sum test, and Kruskal-Wallis test. When a significant difference was found by the Kruskal-Wallis test, the significance of the difference was examined by the Wilcoxon rank-sum test; $p$ values $<0.05$ were considered to indicate statistical significance.

\section{Results}

\section{Study Population}

A total of 109 patients were included in this study (Fig. 1). Among the 49 patients with trauma, 10 (20\%) had penetrating trauma. There were 73 patients who underwent DCL and were managed with TAC using NPWT, including $36(73 \%)$ with trauma and $37(62 \%)$ with intra-abdominal sepsis. Among the 49 trauma patients, injury to the large intestine that required repair or anastomosis was observed in 21 . The site of large intestinal injury was the ascending colon in 33\% (7 patients), the transverse colon in $38 \%$ (8 patients), the descending colon in 14\% (3 patients), the sigmoid colon in $28 \%$ (6 patients) and in 2 
different sites of the large intestine in 3 patients. The site of perforation of the lower digestive tract was the ascending colon in 15\% (9 patients), the transverse colon in $8 \%$ (5 patients), the descending colon in $3 \%$ ( 2 patients), the sigmoid colon in $55 \%$ (33 patients), the rectum in 13 patients (21\%) and several different sites of the large intestine in 2 patients.

The no-DCL group included 9 patients who underwent repair, 8 who underwent colostomy (including 1 who underwent the Miles technique), 7 who underwent small intestine-small intestine anastomosis, 6 who underwent small intestine-colon anastomosis, and 6 who underwent colon-colon anastomosis.

The DCL-early group included 19 patients who underwent repair, 6 who underwent colostomy (including 3 who underwent the Miles technique), 1 who underwent stomach-small intestine anastomosis, 3 who underwent small intestine-small intestine anastomosis, 2 who underwent small intestine-colon anastomosis, 7 who underwent colon-colon anastomosis, and 3 who underwent colon-rectal anastomosis.

During the second-look procedure, the DCL-delay group included 7 patients who underwent ileal stoma, 4 who underwent colostomy, 6 who underwent small intestine-small intestine anastomosis, 5 who underwent small intestine-colon anastomosis, 8 who underwent colon-colon anastomosis, and 2 who underwent colon-rectal anastomosis. Of the 7 patients who underwent ileostomy, 5 required total colectomy.

\section{Clinical Characteristics of the no-DCL and DCL-early, and DCL-delay Patients}

Among the 73 people who were selected to undergo DCL, 41 underwent repair/anastomosis/artificial anus creation during the initial surgery (DCL-early) and 32 underwent anastomosis/artificial anus creation during second-look surgery (DCL-delay). The average injury severity score (ISS) for overall trauma was $24.3 \pm 10$. The average APACHE II score for overall intra-abdominal sepsis was $30.8 \pm 6.7$. A significant difference was observed between the no-DCL and DCL-delay groups during the initial surgery $(p=0.0018)$. In terms of the amount of bleeding during the initial trauma surgery, a significant difference was observed between the no-DCL and DCL-delay groups and between the DCL-early and DCL-delay groups ( $p=0.0002$ and $p=0.0004$, respectively). In terms of the infusion volume 48 hours after surgery, a significant difference was observed between the no-DCL and DCL-early groups, between the no-DCL and DCL-delay groups, and between the DCL-early and DCL-delay groups $(p=0.0001, p<0.0001$, and $p=$ 0.0128 , respectively). There was no difference in the NPWT output or the relaparotomy duration between the DCL-early and DCL-delay groups, and it was possible to achieve PFC in all cases (Table 1).

Anastomotic leak was observed in a total of 6 patients, including 2 in the no-DCL group ( 1 who underwent repair and 1 who underwent artificial anus construction) and 4 who underwent DCL ( 2 who underwent repair and 2 who underwent anastomosis).

Comparison of the Presence/Absence of Complications and Therapeutic Intervention in DCL Patients and the Infusion Volume, NPWT Output, and Urine Volume 
The average infusion volume up to 48 hours from surgery in all patients who underwent DCL was $11246.5 \pm 4305.0 \mathrm{ml}$, the average NPWT output was $1239.9 \pm 712.0 \mathrm{ml}$, and the average urine volume was $2876.6 \pm 2253.1 \mathrm{ml}$. Renal replacement therapy was performed for 19 patients with abdominal sepsis and 8 patients with trauma $(p=0.01)$. All cases of anastomotic leakage occurred in the patients with intra-abdominal sepsis $(p=0.0424)$ (Table 2$)$. There was no difference between patients with trauma and those with intra-abdominal sepsis in the rate of vasopressor infusion, albumin administration, $\mathrm{SSI}$, onset of adhesive intestinal obstruction, and death within 28 days, $(p=0.2616, p=0.5617, p=0.1285, p=$ 0.3749 , and $p=0.081$, respectively) (Table 2 ).

\section{Comparison of the Early-Anastomosis and Delayed- Anastomosis Groups}

Among the patients who underwent DCL, 16 underwent early anastomosis, and 21 underwent delayed anastomosis. There was no difference in terms of age, APACHE II score, or ISS. The delayed-anastomosis group included several patients with colon injury due to trauma $(p=0.0271)$. The surgery duration was significantly shorter in the delayed-anastomosis group $(p=0.0001)$. No difference was observed in infusion volume, NPWT output, or urine volume 48 hours after the initial surgery. There were no differences in SSI, intestinal obstruction, suture failure, or death within 28 days (Table 3 ). The average time until anastomosis in the delayed-anastomosis group was $72.9 \pm 40.9 \mathrm{~min}$.

\section{Comparison of the Patients who Did or Did not Undergo Delayed Anastomosis During Second-Look Surgery}

During second-look surgery performed 48 hours after the initial surgery, anastomosis was performed in 11 patients and postponed in 10 patients. Among the 11 patients who underwent anastomosis during the second-look surgery, 10 underwent a simultaneous procedure to close the abdomen. Regarding the serum albumin value 48 hours after the initial surgery (before second-look surgery), there was no significant difference between the anastomosis group and the group for which anastomosis was postponed (Table 4).

\section{Discussion}

This research showed that there was no difference in the infusion volume and NPWT output volume between early anastomosis and delayed anastomosis during TAC in DCL. The presence or absence of anastomosis during TAC management does not affect NPWT output volume. In patients with delayed anastomosis who received the same infusion volume as the early anastomosis patients, anastomosis and abdominal closure were possible in all cases, and infusion restrictions were not necessary in delayed anastomosis compared with early anastomosis. However, it is necessary to exercise care when performing delayed anastomosis. When a delayed anastomosis planned for 48 hours after initial surgery is complicated, the surgeon must consider postponing anastomosis instead of performing it forcibly. Moreover, the serum albumin value 48 hours after the initial surgery is not a useful indicator of whether delayed anastomosis should be postponed or conducted. 
The drainage of ascites using NPWT during TAC has been shown to be beneficial due to such effects as the reduction of intestinal edema [15] and the lowering of inflammatory mediator levels [21-23]. However, there are also reports that suggest that NPWT output volume is correlated with infusion volume and is higher in fatal cases [24]. When ascites are drained with NPWT, albumin is lost, which is associated with the failure of PFC after TAC [17]. Moreover, hypoalbuminemia is a potential factor for dilutive hypoalbuminemia caused by infusion resuscitation $[25,26]$ and is associated with anastomotic leakage after digestive tract surgery [18-20]. Loftus et al. [17] found that late hypoalbuminemia is associated with failure of PFC. According to that report, no difference was observed in the serum albumin value up to 96 hours after TAC between the group with PFC and the group in which PFC could not be achieved. In this study, no difference in the serum albumin level 48 hours after surgery was observed between the no DCL group, the DCL-early group, and the DCL-delay group. Albumin loss due to NPWT output or dilution due to infusion, differenced between abdominal closure and laparotomy were not related to the albumin value. Additionally, there was no difference in the serum albumin value between the group that underwent delayed anastomosis during second-look surgery and the group in which delayed anastomosis was postponed. From these results, we cannot say that the serum albumin value after 48 hours from the initial surgery is a useful indicator of anastomosis. Considering the limited doses of albumin preparation administered and the low number of suture failures, the administration of exogenous albumin to prevent anastomotic leakage and reduce intestinal edema in cases of delayed anastomosis is not always useful.

Increased NPWT output was observed with vasopressor use. Since the NPWT output is related to prognosis, the NPWT output tended to be large in the group that received vasopressors, with the exception of the 3 patients who died within 28 days $(1513.4 \pm 779.7 \mathrm{ml}$ vs $981.4 \pm 449.3 \mathrm{ml} ; \mathrm{p}=0.0021)$. Norepinephrine is an a-adrenergic agonist that has a strong vasoconstricting action [27], and the possibility that it could reduce NPWT output was also considered. However, vasoconstriction leads to increase in venous reflux [28] and creates a special intra-abdominal environment in the only abdominal pressure is negative pressure, which could cause edema and extravasation of water and may be related to the increase in NPWT output. The selection of delayed anastomosis over early anastomosis did not increase the need for renal replacement therapy or the administration of vasoactive drugs or albumin. These findings show that the financial burden associated with delayed anastomosis is the same as that associated with early anastomosis. Interestingly, the delayed anastomosis group tended to have a shorter laparotomy duration than the early anastomosis group. The financial burden associated with TAC management is a result of delayed anastomosis. Delayed anastomosis involves considerable trauma and may be associated less with intra-abdominal contamination than with intra-abdominal sepsis and persistent inflammation resulting from intra-abdominal sepsis and intestinal edema [29]. The laparotomy duration found in this study is considered to be a localized result in view of bias regarding the disease and that the abdomen was closed at the discretion of the surgeon.

An association between insufficient infusion and NPWT output volume has been indicated as a reason for acute kidney injury [30]. In this study, it was observed that the NPWT output volume tended to be large in the group that received renal replacement therapy. No difference was observed in the infusion volume, but the urine volume differed. Renal damage is considered to be the reason for the suppressed urine 
volume and large NPWT output. However, the possibility that increased NPWT output and insufficient infusion led to the occurrence of renal damage cannot be ruled out. A bolus administration of infusion corresponding to the NPWT output volume could have prevented the need for renal replacement therapy. In NPWT management, the optimal negative pressure treatment to maximize tissue growth is approximately $-125 \mathrm{mmHg}$, and the pressure level when active bleeding due to coagulative failure is suspected is approximately $-75 \mathrm{mmHg}[31,32]$. Loftus et al. $[16,17,29,33]$ reported that $1300-1900 \mathrm{ml}$ was the median value of NPWT output up to 48 hours after TAC in 4 studies that reported NPWT output. However, this could not be confirmed except in 1 report in which the NPWT negative pressure (suction amount) was $-75 \mathrm{mmHg}$. In this study, the median value of NPWT output up to 48 hours after surgery was $1080 \mathrm{ml}$. The NPWT output was less than that in the previously mentioned report. Our suction pressure setting was $-30 \mathrm{cmH} 2 \mathrm{O}(1 \mathrm{mmHg}=1.36 \mathrm{cmH} 20)$, and low pressure management was considered one of the factors for this comparably low output. This study showed that the presence or absence of anastomosis does not impact NPWT ejection volume; however, since the NPWT output volume is predicted to depend on the suction pressure, it is necessary to consider the difference in suction pressure.

The NPWT output was lower, and the urine volume tended to be higher, in the group that underwent anastomosis during second-look surgery than in the group with postponed anastomosis. Sustainable maintenance of urine volume is considered to have reduced NPWT output, and the suppression of intestinal edema made it possible to perform anastomosis. The group in which anastomosis was postponed had higher physiological severity and more frequently received renal replacement therapy. There was no protocol for postponing delayed anastomosis, and the decision was made at the discretion of the surgeon. In the group with postponed anastomosis, the PaO2/FiO2 ratio before the second-look surgery tended to be lower, and CRP tended to be high, and general conditions and inflammation may have been involved in the postponement of anastomosis. Regarding delayed anastomosis after 48 hours, the large NPWT output may be associated with the postponement of anastomosis reflecting persistent inflammation, intestinal edema and general conditions. Additionally, low NPWT output is not necessarily disadvantageous for delayed anastomosis. The effect of drainage due to NPWT output over 48 hours must be comprehensively evaluated in combination with other body fluid balance indicators. It is difficult to think of PFC and anastomosis as the same procedure since their purpose, time to implementation and such differ. When the ultimate goal is anastomosis, early anastomosis and delayed anastomosis can be managed with the same infusion volume. However, in terms of whether delayed anastomosis is conducted or postponed, the infusion volume is related to pathology and hence should be the subject of further study by disease with a greater number of subjects.

In this study, the delayed anastomosis group included many cases of large intestine damage due to trauma, and bias was observed. Possible reasons are as follows: In large intestine injury, there is no protocol for selecting delayed anastomosis, and the surgeon may have consciously avoided selecting patients with factors such as dislodging of the colon (mobilization) for reconstruction, etc., that may prolong the duration of surgery or cause unnecessary bleeding. In $\mathrm{HVI}$ and lower digestive tract perforation, controlling the leakage of intestinal contents must be considered. Even in cases in which the 
abdomen can be physically closed, the advantage of selecting open abdomen as a drainage effect corresponding to intra-abdominal contamination has been shown [34]. The purpose of performing early anastomosis during the initial surgery and selecting TAC is to move the patient to intensive care with the aim of controlling rapid bleeding and contamination, shorten the surgery time to greatest the possible extent, and encourage early physiological optimization [35]. However, it is necessary to reconsider some matters, such as the need for TAC with early anastomosis and whether patients for whom early anastomosis is possible might not have been selected for delayed anastomosis. Regarding DCL, overuse [36] and increased risk of abdominal complications [37] have also been pointed out. In addition, it is necessary to recognize that delayed anastomosis itself can have negative impacts; reports have described it as a disadvantageous treatment strategy [38] and have raised the possibility of ongoing peritonitis [39], increased anastomotic leakage if PFC is not possible during second-look surgery [40], and other factors.

This study has several limitations. First, our data were obtained from a single center with a limited number of diseases and cases. Second, the albumin value included in the ascites drained from NPWT was not measured, and therefore, the amount of albumin lost could not be clarified. Third, there are no clear criteria regarding the decision to perform or postpone delayed anastomosis. It is necessary to establish objective treatment selection criteria that do not depend on the subjective judgment of the surgeon. In the future, reconsideration to address multiple breaking points is required.

\section{Conclusions}

Compared with patients who underwent early anastomosis, those for whom delayed anastomosis was selected during DCL do not necessarily require infusion restrictions for the purpose of anastomosis. The presence or absence of anastomosis during TAC management does not affect the NPWT output volume, and both early anastomosis and delayed anastomosis can be managed with the same infusion volume. However, in cases in which the planned delayed anastomosis is complicated, it is necessary to postpone anastomosis rather than perform it.

\section{Abbreviations}

DCL

Damage control laparotomy

NPWT

Negative pressure wound therapy

PFC

Primary fascial closure

HVI

Hollow viscous injury

MI

Mesenteric injury 
TAC

Temporary abdominal closure

\section{Declarations}

\section{Ethical Approval and Consent to Participate}

The protocol of the study was approved by the Iwate Medical University review board (MH2018-611). Consent to participate is not applicable because the data sets were pseudonymous and this study was retrospective.

\section{Consent for Publication}

Not applicable

\section{Availability of Data and Materials}

All relevant data are presented in the published manuscript.

\section{Competing Interests}

The authors declare that they have no competing interests.

\section{Funding}

Not applicable.

\section{Authors' Contributions}

YT, SS, MK designed the study.YT, AK, SS, YN, HS, KI collected the patient's clinical data. YT, MK contributed to wrote the paper. $\mathrm{YN}, \mathrm{SE}, \mathrm{YI}, \mathrm{AS}$ contributed to data interpretation, and manuscript drafting. YT, SS, HS, MK contributed to the statistical analysis and data interpretation. AS contributed to manuscript critical revision. All authors read and approved the final manuscript.

\section{Acknowledgements}

We thank American Journal Experts (https://www.aje.com) for English language editing.

\section{Authors' Information}

\section{Affiliations}

Department of Surgery, Iwate Medical University, School of Medicine, Iwate, Japan

Yohta Tanahashi, Akiko Kawakami, Shusaku Sasaki, Yu nishinari, Kaoru Ishida, Masahiro Kojika, Akira Sasaki 
Department of Critical Care Medicine, Iwate Medical University, School of Medicine, Iwate, Japan

Hisaho Sato, Masahiro Kojika, Shigeatsu Endo, Yoshihiro Inoue

Morioka Yuai Hospital, Morioka, Iwate, Japan

Shigeatsu Endo

\section{Corresponding Author}

Masahiro Kojika

\section{References}

1. Stone HH, Strom PR, Mullins RJ. Management of the major coagulopathy with onset during laparotomy. Ann Surg. 1983;197:532-5.

2. Rotondo MF, Schwab CW, McGonigal MD, Phillips GR 3rd, Fruchterman TM, Kauder DR, et al. 'Damage control': an approach for improved survival in exsanguinating penetrating abdominal injury. J Trauma. 1993;35:375 - 82; discussion 82 - 3..

3. Miller PR, Chang MC, Hoth JJ, Holmes JHT, Meredith JW. Colonic resection in the setting of damage control laparotomy: is delayed anastomosis safe? Am Surg. 2007;73:606-9; discussion 9-10.

4. Ordoñez CA, Pino LF, Badiel M, Sánchez Al, Loaiza J, Ballestas L, et al. Safety of performing a delayed anastomosis during damage control laparotomy in patients with destructive colon injuries. $J$ Trauma. 2011;71:1512-7; discussion 7-8.

5. Sharpe JP, Magnotti LJ, Fabian TC, Croce MA. Evolution of the operative management of colon trauma. Trauma Surg Acute Care Open. 2017;2:e000092.

6. Tatebe LC, Jennings A, Tatebe K, Handy A, Prajapati P, Smith M, et al. Traumatic colon injury in damage control laparotomy-a multicenter trial: is it safe to do a delayed anastomosis? J Trauma Acute Care Surg. 2017;82:742-9.

7. Ferrara A, MacArthur JD, Wright HK, Modlin IM, McMillen MA. Hypothermia and acidosis worsen coagulopathy in the patient requiring massive transfusion. Am J Surg. 1990;160:515-8.

8. Rotondo MF, Zonies DH. The damage control sequence and underlying logic. Surg Clin North Am. 1997;77:761-77.

9. Moore EE, Burch JM, Franciose RJ, Offner PJ, Biffl WL. Staged physiologic restoration and damage control surgery. World J Surg. 1998;22:1184-90. discussion $90-1$.

10. Perathoner A, Klaus A, Mühlmann G, Oberwalder M, Margreiter R, Kafka-Ritsch R. Damage control with abdominal vacuum therapy (VAC) to manage perforated diverticulitis with advanced generalized peritonitis-a proof of concept. Int J Colorectal Dis. 2010;25:767-74.

11. Sohn M, lesalnieks I, Agha A, Steiner P, Hochrein A, Pratschke J, et al. Perforated diverticulitis with generalized peritonitis: low stoma rate using a "damage control strategy". World J Surg. 
2018;42:3189-95.

12. Tartaglia D, Costa G, Camillò A, Castriconi M, Andreano M, Lanza M, et al. Damage control surgery for perforated diverticulitis with diffuse peritonitis: saves lives and reduces ostomy. World J Emerg Surg. 2019;14:19.

13. Smith JW, Garrison RN, Matheson PJ, Franklin GA, Harbrecht BG, Richardson JD. Direct peritoneal resuscitation accelerates primary abdominal wall closure after damage control surgery. J Am Coll Surg. 2010;210:658-64, 664-7.

14. Huang Q, Zhao R, Yue C, Wang W, Zhao Y, Ren J, et al. Fluid volume overload negatively influences delayed primary facial closure in open abdomen management. J Surg Res. 2014;187:122-7.

15. Tian W, Huang Q, Yao Z, Huang M, Yang F, Zhao Y, et al. A preliminary prospective study of patients who underwent vacuum-assisted and mesh-mediated fascial traction techniques for open abdomen management with negative fluid therapy: an observational study. Med (Baltim). 2019;98:e16617.

16. Loftus TJ, Efron PA, Bala TM, Rosenthal MD, Croft CA, Walters MS, et al. The impact of standardized protocol implementation for surgical damage control and temporary abdominal closure after emergent laparotomy. J Trauma Acute Care Surg. 2019;86:670-8.

17. Loftus TJ, Jordan JR, Croft CA, Smith RS, Efron PA, Moore FA, et al. Characterization of hypoalbuminemia following temporary abdominal closure. J Trauma Acute Care Surg. 2017;83:6506.

18. Lai CC, You JF, Yeh CY, Chen JS, Tang R, Wang JY, et al. Low preoperative serum albumin in colon cancer: a risk factor for poor outcome. Int J Colorectal Dis. 2011;26:473-81.

19. Parthasarathy M, Greensmith M, Bowers D, Groot-Wassink T. Risk factors for anastomotic leakage after colorectal resection: a retrospective analysis of 17518 patients. Colorectal Dis. 2017;19:28898.

20. Huang J, Zhou Y, Wang C, Yuan W, Zhang Z, Chen B, et al. Logistic regression analysis of the risk factors of anastomotic fistula after radical resection of esophageal-cardiac cancer. Thorac Cancer. 2017;8:666-71.

21. Kubiak BD, Albert SP, Gatto LA, Snyder KP, Maier KG, Vieau CJ, et al. Peritoneal negative pressure therapy prevents multiple organ injury in a chronic porcine sepsis and ischemia/reperfusion model. Shock. 2010;34:525-34.

22. Roberts DJ, Zygun DA, Grendar J, Ball CG, Robertson HL, Ouellet JF, et al. Negative-pressure wound therapy for critically ill adults with open abdominal wounds: a systematic review. J Trauma Acute Care Surg. 2012;73:629-39.

23. Hu P, Uhlich R, Gleason F, Kerby J, Bosarge P. Impact of initial temporary abdominal closure in damage control surgery: a retrospective analysis. World J Emerg Surg. 2018;13:43.

24. Cheatham ML, Demetriades D, Fabian TC, Kaplan MJ, Miles WS, Schreiber MA, et al. Prospective study examining clinical outcomes associated with a negative pressure wound therapy system and Barker's vacuum packing technique. World J Surg. 2013;37:2018-30. 
25. Ryan AM, Hearty A, Prichard RS, Cunningham A, Rowley SP, Reynolds JV. Association of hypoalbuminemia on the first postoperative day and complications following esophagectomy. $J$ Gastrointest Surg. 2007;11:1355-60.

26. Hübner M, Mantziari S, Demartines N, Pralong F, Coti-Bertrand P, Schäfer M. Postoperative albumin drop is a marker for surgical stress and a predictor for clinical outcome: a pilot study. Gastroenterol Res Pract. 2016;2016:8743187.

27. Martin C, Papazian L, Perrin G, Saux P, Gouin F. Norepinephrine or dopamine for the treatment of hyperdynamic septic shock? Chest. 1993;103:1826-31.

28. Persichini R, Silva S, Teboul JL, Jozwiak M, Chemla D, Richard C, et al. Effects of norepinephrine on mean systemic pressure and venous return in human septic shock. Crit Care Med. 2012;40:3146-53.

29. Loftus TJ, Jordan JR, Croft CA, Smith RS, Efron PA, Mohr AM, et al. Temporary abdominal closure for trauma and intra-abdominal sepsis: different patients, different outcomes. J Trauma Acute Care Surg. 2017;82:345-50.

30. Loftus TJ, Bihorac A, Ozrazgat-Baslanti T, Jordan JR, Croft CA, Smith RS, et al. Acute kidney injury following exploratory laparotomy and temporary abdominal closure. Shock. 2017;48:5-10.

31. Chiara O, Cimbanassi S, Biffl W, Leppaniemi A, Henry S, Scalea TM, et al. International consensus conference on open abdomen in trauma. J Trauma Acute Care Surg. 2016;80:173 - 83.

32. Chabot E, Nirula R. Open abdomen critical care management principles: resuscitation, fluid balance, nutrition, and ventilator management. Trauma Surg Acute Care Open. 2017;2:e000063.

33. Loftus TJ, Efron PA, Bala TM, Rosenthal MD, Croft CA, Smith RS, et al. Hypertonic saline resuscitation after emergent laparotomy and temporary abdominal closure. J Trauma Acute Care Surg. 2018;84:350-7.

34. Kirkpatrick AW, Coccolini F, Ansaloni L, Roberts DJ, Tolonen M, McKee JL, et al. Closed or open after source control laparotomy for severe complicated intra-abdominal sepsis (the COOL trial): study protocol for a randomized controlled trial. World J Emerg Surg. 2018;13:26.

35. Germanos S, Gourgiotis S, Villias C, Bertucci M, Dimopoulos N, Salemis N. Damage control surgery in the abdomen: an approach for the management of severe injured patients. Int J Surg. 2008;6:24652.

36. Hatch QM, Osterhout LM, Podbielski J, Kozar RA, Wade CE, Holcomb JB, et al. Impact of closure at the first take back: complication burden and potential overutilization of damage control laparotomy. J Trauma. 2011;71:1503-11.

37. George MJ, Adams SD, McNutt MK, Love JD, Albarado R, Moore LJ, et al. The effect of damage control laparotomy on major abdominal complications: a matched analysis. Am J Surg. 2018;216:56-9.

38. Ott MM, Norris PR, Diaz JJ, Collier BR, Jenkins JM, Gunter OL, et al. Colon anastomosis after damage control laparotomy: recommendations from 174 trauma colectomies. J Trauma. 2011;70:595-602.

39. Sohn MA, Agha A, Steiner P, Hochrein A, Komm M, Ruppert R, et al. Damage control surgery in perforated diverticulitis: ongoing peritonitis at second surgery predicts a worse outcome. Int $\mathrm{J}$ 
Colorectal Dis. 2018;33:871-8.

40. Anjaria DJ, Ullmann TM, Lavery R, Livingston DH. Management of colonic injuries in the setting of damage-control laparotomy: one shot to get it right. J Trauma Acute Care Surg. 2014;76:594-8; discussion 598-600.

\section{Tables}

Due to technical limitations, tables 1 to 4 is only available as a download in the Supplemental Files section.

\section{Figures}

Fig. 1

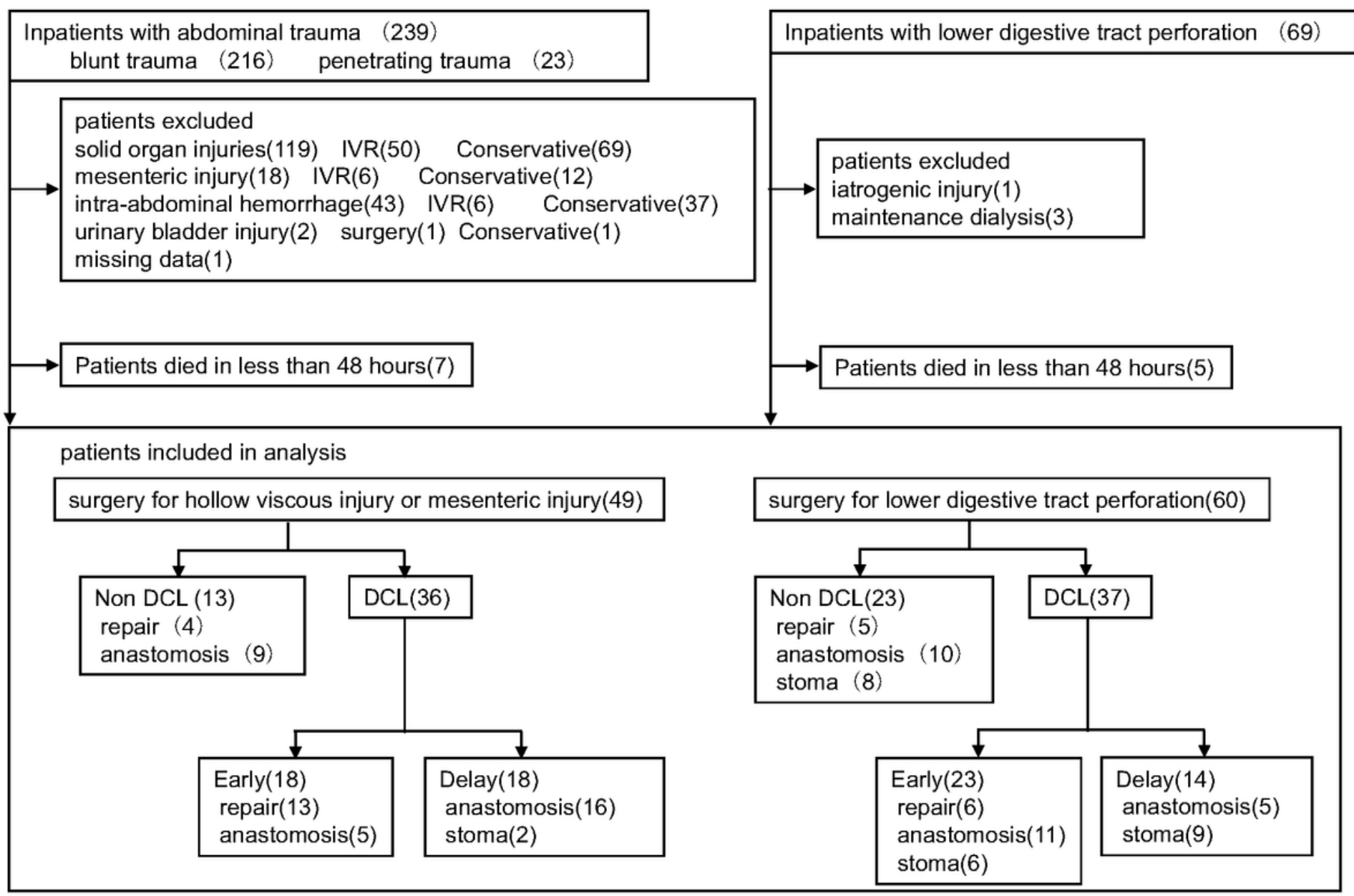

Study flowchart, DCL:Damage control laparotomy

\section{Figure 1}

Study flowchart, DCL: Damage control laparotomy 


\section{Supplementary Files}

This is a list of supplementary files associated with this preprint. Click to download.

- table1.xlsx

- table2.xIsx

- table3.xIsx

- table4.xIsx 